\title{
PRODUÇÃO DE MUDAS MICORRIZADAS DE Schinus terebinthifolius Raddi. EM DIFERENTES SUBSTRATOS
}

\author{
Rodrigo Camara de Souza ${ }^{1}$, Marcos Gervásio Pereira ${ }^{2}$, Rômulo Guimarães Giácomo ${ }^{3}$ \\ Eliane Maria Ribeiro da Silva ${ }^{4}$, Luís Fernando Tavares de Menezes ${ }^{5}$
}

\author{
${ }^{1}$ Eng. Agrônomo, M.Sc., Doutorando em Ecologia e Recursos Naturais, UENF, Seropédica, RJ, Brasil - rcamara73@ gmail.com \\ ${ }^{2}$ Eng. Agrônomo, Dr., Depto. de Solos, UFRRJ, Seropédica, RJ, Brasil - gervasio@ufrrj.br \\ ${ }^{3}$ Mestrando em Ciências Ambientais e Florestais, UFRRJ, Seropédica, RJ, Brasil \\ ${ }^{4}$ Eng $^{\mathrm{a}}$. Florestal, Dr ${ }^{\mathrm{a}}$, EMBRAPA Agrobiologia, Seropédica, RJ, Brasil \\ ${ }^{5}$ Biólogo, Dr., Depto. de Ciências da Saúde, Biológicas e Agrárias, UFES, São Mateus, ES, Brasil
}

Recebido para publicação: 17/01/2008 - Aceito para publicação: 22/06/2008

\begin{abstract}
Resumo
Avaliou-se a influência de dois tipos de substrato (S1 e S2), na ausência e na presença de inoculação de fungos micorrízicos arbusculares (FMA), na produção de mudas de Schinus terebinthifolius em tubetes de $280 \mathrm{~mL}$. O experimento foi realizado em casa de vegetação do Departamento de Solos do Instituto de Agronomia, Universidade Federal Rural do Rio de Janeiro, Seropédica, RJ, no período de junho a setembro de 2005. S1 e S2 se constituíram numa mistura (em volume), previamente desinfestada, de fosfato de rocha (10\%), areia grossa lavada (30\%), material argiloso (30\%) e matéria orgânica (30\%). Para S1, a fonte de matéria orgânica foi resíduo industrial de produção de borracha sintética (LETRIP), enquanto que para S2, esterco bovino curtido. O delineamento experimental foi o inteiramente casualizado, em esquema de parcelas subdivididas, com 24 repetições, com 6 plantas por parcela. Aos 120 dias após a montagem do experimento, observou-se que $\mathrm{S} 2$ proporcionou às mudas ganhos significativos em praticamente todas as variáveis analisadas (altura, diâmetro, massa seca de parte aérea e de raiz, teor de N, P e K na parte aérea, e de $\mathrm{N}$ na raiz), em comparação com S1. Não houve diferença significativa entre os substratos quanto à ausência e presença de FMA, possivelmente em função dos elevados teores de nutrientes em S1 e S2. A esse mesmo fato se atribuiu a ausência de colonização de raízes por FMA em S2. O LETRIP desempenhou efeito protetor-encapsulador de esporos de FMA nativos contra a ação do fungicida, o que permitiu a colonização de raízes em S1 na ausência de inoculação. Portanto, S2 mostrou ser mais indicado para a produção de mudas de S. terebinthifolius.

Palavras-chave: Produção de mudas florestais; micorriza; áreas degradadas.
\end{abstract}

\begin{abstract}
Schinus terebinthifolius Raddi. seedlings production with arbuscular mycorrhizal fungi in differents substrates. The present work aimed to evaluate the effetc of two types of substratum (S1 and S2), in the presence or absence of arbuscular mycorhizal fungi (AMF), on Schinus terebinthifolius seedlings, using tubes of $280 \mathrm{~mL}$. The experiment was carried out in greenhouse at Departamento de Solos, Instituto de Agronomia, Universidade Federal Rural do Rio de Janeiro, Seropédica, RJ, to June from September 2005. S1 and S2 were constituted by a mixture (in volume), previously disinfested, of rocks's fosfato (10\%), washed thick sand (30\%), clay (30\%) and organic matter $(30 \%)$. For S1, the source of organic matter was industrial residue of synthetic rubber production (LETRIP), whereas for S2, bovine manure. The experimental design was completely randomized in a split-plot with 24 replicates and 6 plants per plot. At 120 days after experiment instalation, S2 showed to be superior than $\mathrm{S} 1$ for most of the datas (height, diameter, shoot and root dry mass, N, P and $\mathrm{K}$ shoot concentrations, and $\mathrm{N}$ root concentration). There wasn't any significant difference between the inoculated and not inoculated treatments, probably because of the high nutrients concentration in S1 and S2. And so it was the reason of the absence of root colonization in S2. LETRIP played a protector effect in native AMF spores against fungicide action, which permited root colonization in S1 without inoculation. In conclusion, S2 was more indicated to $S$. terebinthifolius seedling production.

Keywords: Forestry seedlings production; mycorrhizal fungi; disturbed areas.
\end{abstract}




\section{INTRODUÇÃO}

A derrubada das florestas para a instalação de atividade agropecuária ou de reflorestamento comercial, a urbanização e a industrialização, entre outros fatores, são responsáveis pelo aumento crescente de áreas degradadas e paisagens fragmentadas e, consequentemente, pela redução da biodiversidade e pelo aumento do risco de extinção de espécies (KAGEYAMA; GANDARA, 2005). Desse panorama surge a necessidade de se produzirem mudas de essências florestais para suprir a demanda de programas de recomposição da vegetação nessas áreas (CARVALHO FILHO et al., 2003), cuja preocupação tem envolvido o uso de espécies nativas regionais, as quais são supostamente mais adaptadas às condições edafoclimáticas locais e, desse modo, facilitariam o restabelecimento da vegetação e de suas relações com a fauna (CARRASCO; CASTANHEIRA, 2004).

Aproximadamente $80 \%$ das espécies das restingas do estado do Rio de Janeiro ocorrem tanto na Mata Atlântica quanto nas restingas (ARAÚJO, 2000), o que corrobora a hipótese de que a flora das restingas é originária principalmente da Mata Atlântica (RIZZINI, 1997). Dessa forma, a produção de mudas de espécies de restingas é interessante para atender a planos de recuperação que envolvam a Mata Atlântica, de uma maneira geral.

A falta de mudas nativas e os elevados custos de produção inibem as iniciativas de projetos de restauração ambiental, que podem ser popularizadas com o conhecimento sobre a maneira adequada de se produzirem mudas de essências florestais (germinação, repicagem ou semeadura direta, embalagem, substrato, luminosidade) e sobre a redução desse custo (JESUS; ROLIM, 2005).

O desenvolvimento de protocolos e estratégias para a produção de mudas com qualidade, em menor tempo e em condições acessíveis é muito importante (CARRASCO; CASTANHEIRA, 2004; CUNHA et al., 2005). Dessa maneira, estudos envolvendo substratos alternativos e recipientes adequados para baratear os custos de produção e a inoculação das mudas com fungos micorrízicos arbusculares (SAGGIN JÚNIOR; SILVA, 2005), microrganismos que favorecem o estabelecimento das mudas nas áreas degradadas (FRANCO et al., 1992), são de grande valia. Nesse âmbito, cabem pesquisas voltadas para o emprego de resíduos industriais como constituintes de substratos na produção de mudas florestais, uma vez que alguns resíduos, em função de seus atributos físico-químicos, podem atuar como corretivos da acidez, fornecer nutrientes às plantas e melhorar as propriedades físicas e químicas do solo, como fruto do aumento do teor de matéria orgânica (DIAS, 2005), além de que essa via de utilização pode contribuir sobremaneira com o aproveitamento econômico-ambiental adequado de tais materiais, pois, de acordo com Dias et al. (2004), a disposição final dos resíduos industriais configurou-se num desafio para as indústrias e para a sociedade.

Alguns estudos comprovaram a efetividade do emprego do LETRIP, lodo da estação de tratamento de resíduos industriais da Petroflex, em atividades agrícolas e florestais. No cultivo de soja, a adição do referido resíduo ao solo ocasionou elevação significativa de $\mathrm{pH}$ e dos teores de potássio e fósforo, além do aumento significativo do rendimento do grão da soja (DIAS, 2005), enquanto que, como constituinte do substrato para a produção de mudas de Eucalyptus saligna Smith, foi responsável pelo aumento de massa seca da parte aérea das plantas (MALHEIROS et al., 2005).

Os fungos micorrízicos arbusculares (FMA) são micro-organismos biotróficos obrigatórios que se associam a raízes secundárias da planta hospedeira (CARNEIRO et al., 1998), promovendo um aumento do volume de solo explorado pelo sistema radicular. Assim sendo, desempenham importante papel na ciclagem de nutrientes, principalmente nos trópicos, pois impedem uma rápida perda por lixiviação e permitem sua conservação nesses ecossistemas, onde grande parte dos nutrientes disponíveis se concentra na biomassa (ODUM, 1988). Portanto, a inoculação de mudas florestais com FMA pode otimizar a obtenção de nutrientes, principalmente fósforo, pelas plantas, sem que seja necessário lançar mão de grandes quantidades de adubo, o que contribui para diminuir o custo total de produção de mudas. Entretanto, a eficiência simbiótica pode ser comprometida se o nível de fósforo for muito baixo na solução do solo (SOUZA; SILVA, 1996), o que comumente ocorre nos solos tropicais. Além do mais, espécies pioneiras são mais responsivas ao fornecimento de fósforo, indicando a necessidade do suprimento adequado desse nutriente para o seu desenvolvimento (RESENDE et al., 1999). Então, mesmo inoculando as mudas, é necessária a aplicação de doses econômicas de adubo fosfatado no processo de produção das mudas e no plantio no campo. 
Schinus terebinthifolius Raddi., popularmente conhecida como aroeira-pimenteira, varia de arbusto a árvore perenifólia com dois a dez metros de altura (CARVALHO, 2003). É uma Anacardiaceae nativa do Brasil que cresce também em terrenos secos e pobres e ocorre de Pernambuco até o Rio Grande do Sul e Mato Grosso do Sul. Devido a sua ampla dispersão, é encontrada em várias formações vegetais, desde a restinga até as florestas pluvial e semidecídua de altitude (LORENZI, 2000). Na Restinga da Marambaia (RJ), é uma espécie muito comum na face das dunas voltada para o oceano (MENEZES et al., 1998), onde imprime um caráter lenhoso (CARVALHO, 2003). É considerada pioneira (LORENZI, 2000), o que a torna bioindicadora do caráter edáfico dos ambientes naturais ou antropizados (LENZI e ORTH, 2004) e indica seu grande potencial de crescimento (GONÇALVES et al., 2003), o qual acelera a formação de um hábitat mais adequado à recolonização da área degradada por outras espécies, favorecendo a sucessão ecológica.

O presente estudo teve como objetivo testar dois tipos de substrato, na ausência ou presença de fungos micorrízicos arbusculares, para a produção de mudas de Schinus terebinthifolius.

\section{MATERIAL E MÉTODOS}

Em um experimento iniciado em junho de 2005, em casa de vegetação do Departamento de Solos do Instituto de Agronomia da Universidade Federal Rural do Rio de Janeiro, Seropédica, RJ, foram testados dois tipos de substratos, ambos na presença e na ausência de FMA, para a produção de mudas de Schinus terebinthifolius em tubetes plásticos de $280 \mathrm{ml}$ (S1 e S2). Ambos foram obtidos pela mistura, em volume, na proporção 10:30:30:30, dos seguintes constituintes: 100 g.dm ${ }^{-3}$ de fosfato de rocha (Fosfato de Araxá), 300 g.dm ${ }^{-3}$ de areia grossa lavada e peneirada, $300 \mathrm{~g}^{-\mathrm{dm}^{-3}}$ de material coletado em um horizonte B de um Argissolo Vermelho-Amarelo com $50 \%$ de argila e $300 \mathrm{~g}_{\mathrm{dm}} \mathrm{dm}^{-3} \mathrm{de}$ matéria orgânica. A diferença entre eles residiu no fato de que este último componente do substrato empregado em S1 foi LETRIP (lodo da estação de tratamento de resíduos industriais da Petroflex), um resíduo proveniente da produção de borracha sintética pela Petroflex Indústria e Comércio S.A., considerado não-inerte (Classe II), segundo a NBR 10.007. Já em S2, a fonte de matéria orgânica empregada foi esterco bovino de curral curtido.

A tabela 1 traz os resultados da análise química dos componentes dos substratos, que foi realizada de acordo com Embrapa (1997). De acordo com o Manual de Adubação para o Estado do Rio de Janeiro (FREIRE et al., 1988), o LETRIP apresentou alto teor disponível de $\mathrm{Ca}^{++}+\mathrm{Mg}^{++}$, enquanto o esterco, médio teor. Em ambos os componentes, o teor disponível de $\mathrm{P}$ e $\mathrm{K}$ encontrado foi considerado muito alto, sendo notável o de P em LETRIP e o de K no esterco, o que, em comparação com o alto custo dos adubos industrializados fornecedores desses nutrientes, justifica o uso de ambos na produção de mudas.

Tabela 1. Características químicas dos componentes dos substratos, para a produção de mudas de Schinus terebinthifolius Raddi.

Table 1. Chemical characteristics of the substrates components used for Schinus terebinthifolius Raddi. seedlings production.

\begin{tabular}{|c|c|c|c|c|c|c|c|}
\hline \multirow{2}{*}{$\begin{array}{l}\text { Componentes } \\
\text { substrato }\end{array}$} & pH em água & $\mathrm{Al}^{+3}$ & $\mathrm{Ca}^{+2}+\mathrm{Mg}^{+2}$ & $\overline{\mathrm{Ca}^{+2}}$ & $\overline{\mathrm{Mg}^{+2}}$ & $\mathbf{P}$ & $\mathbf{K}^{+}$ \\
\hline & & \multicolumn{4}{|c|}{$\left(\mathrm{cmol}_{\mathrm{c}} \mathrm{dm}^{-3}\right)$} & \multicolumn{2}{|c|}{$\left(\mathrm{mg.dm}{ }^{-3}\right)$} \\
\hline Argila & 4,7 & 0,4 & 0,3 & 0,2 & 0,1 & * & 47,4 \\
\hline LETRIP & 6,6 & 0,1 & 7,0 & 5,3 & 1,7 & 2268,0 & 727,0 \\
\hline Esterco & 7,4 & 0,1 & 2,6 & 1,3 & 1,3 & 294,8 & 2928,0 \\
\hline
\end{tabular}

* teor não-detectável.

Em betoneira, os substratos foram homogeneizados separadamente e desinfestados com aplicação do fungicida benomyl (formulação pó molhável 50\%), na dosagem de $20 \mathrm{mg} \cdot \mathrm{kg}^{-1} \mathrm{de}$ substrato, conforme preconizado por Kahiluoto e Vestberg (2000). Em seguida, os substratos foram umedecidos e armazenados em sacos plásticos por três dias. Ao final desse período, os substratos foram cobertos por lona plástica preta e, na casa de vegetação onde o experimento foi conduzido, foram deixados secar ao ar livre, por sete dias. 
Cada tubete, em um orifício de $1 \mathrm{~cm}$ de profundidade, recebeu quatro sementes e, nos tratamentos que receberam inoculações com fungos micorrízicos, foi adicionada uma medida padrão contendo uma mistura equilibrada com esporos das espécies Gigaspora margarita Becker \& Hall e Glomus clarum Nicolson \& Schenk, produzida em cultura-estoque de Brachiaria decumbens Staft., que depois foi coberta com o respectivo substrato. Para equilibrar a microbiota dos tratamentos, foram adicionados a cada tubete $2 \mathrm{~mL}$ de um filtrado do inoculante micorrízico, isento de propágulos desses micro-organismos, preparado com o processamento de aproximadamente $40 \mathrm{~g}$ do inóculo com $400 \mathrm{~mL}$ de água destilada, em liquidificador. O material triturado foi passado uma vez por peneira de $0,053 \mathrm{~mm}$ e duas vezes por papel de filtro. Após a adição do filtrado, os vasos foram irrigados, para facilitar a penetração desse produto nos poros do substrato.

O delineamento experimental adotado foi inteiramente casualizado, em esquema de parcelas subdivididas, com 24 repetições. Nas parcelas foram avaliados os dois condicionantes de substrato, ao passo que, nas subparcelas, a presença e a ausência do inóculo com FMA, totalizando tratamentos: S1 sem inoculação com FMA; S1 com inoculação com FMA; S2 sem inoculação; S2 com inoculação.

Quando as plântulas apresentaram um par de folhas definitivas, foi realizado o desbaste, deixando-se apenas uma planta por tubete e, aos 120 dias após a montagem do experimento, foram avaliadas a altura, com régua centimetrada, e o diâmetro à altura do coleto das mudas, com paquímetro digital. Em seguida, o material foi fracionado em parte aérea e raiz. O sistema radicular foi lavado em água destilada e em seguida colocado em papel absorvente para retirar-se o excesso de umidade. Para a avaliação da colonização dos fungos micorrízicos, separou-se $0,5 \mathrm{~g}$ de raízes finas $(<1,00 \mathrm{~mm}$ de diâmetro) localizadas junto ao substrato. Esse material foi conservado em etanol 50\%, para o posterior clareamento e coloração, conforme as metodologias propostas por Koske e Gemma (1989) e Grace e Stribley (1991). As raízes, lavadas com água corrente, permaneceram cobertas com solução de KOH a 2,5\%, por 24 horas. Em seguida, foram novamente lavadas em água corrente e permaneceram imersas em $\mathrm{H}_{2} \mathrm{O}_{2}$ alcalina a $3 \%$, por aproximadamente 30 minutos. O material passou por nova lavagem com água corrente e foi coberto com $\mathrm{HCl} 1 \%$, por 24 horas. Removeu-se o $\mathrm{HCl}$, sem lavar o material, que, depois, foi corado com solução de azul de metila. Por fim, as raízes foram armazenadas em solução ácida de glicerol claro, até o momento da avaliação.

A porcentagem do comprimento de raízes finas colonizadas (COLONRAIZ) foi avaliada pelo método da interseção em placa quadriculada, descrito no trabalho de Giovanetti; Mosse (1980), adaptado a partir do método de medida de comprimento de raízes de Newman (1966), citados por Hungria e Araújo (1994).

O restante do material radicular, assim como a parte aérea, passou por secagem em estufa de circulação de ar forçado a $65{ }^{\circ} \mathrm{C}$, por 48 horas, e pesagem até atingir massa constante, para determinação da massa seca da parte área e a das raízes. Posteriormente, todo o material foi moído para a realização da análise do teor de N, P e K presentes no extrato da raiz e da parte aérea das plantas (TEDESCO et al., 1995).

Para avaliação do número total de esporos dos FMA nos tratamentos, foram empregados 50 $\mathrm{cm}^{3}$ de terra, previamente seca à sombra, para proceder a extração dos mesmos, conforme a técnica do peneiramento úmido (GERDEMANN; NICOLSON, 1963), utilizando-se peneiras com malhas de 38 $\mu \mathrm{m}$, seguida por centrifugação em sacarose. Uma vez extraídos, foi realizada a contagem, com o auxílio de microscópio estereoscópico.

Os resultados de ambos os testes descritos neste capítulo foram submetidos à análise de variância, com a aplicação do teste $\mathrm{F}$ de Snedecor, e à análise das médias, com aplicação do teste de Scott-Knott a 5\% de probabilidade. Esses testes e análises foram realizados com o auxílio do software SAEG-5.0 (Sistema de Análises Estatísticas e Genéticas - Universidade Federal de Viçosa). Na avaliação da homogeneidade das variâncias, foi empregado o teste de Cochrane-Bartlett, e, na análise da normalidade, o teste de Lilliefors.

\section{RESULTADOS E DISCUSSÃO}

De posse dos dados da tabela 1, notou-se que o LETRIP, em comparação com o esterco bovino, apresentou maior disponibilidade de $\mathrm{Ca}, \mathrm{Mg}$ e $\mathrm{P}$ e menor valor de $\mathrm{pH}$. A disponibilidade de micronutrientes é fortemente influenciada pelo $\mathrm{pH}$, pelas condições de óxido-redução, pela matéria 
orgânica e pela presença de certos íons (MALAVOLTA et al., 1991). Assim sendo, estima-se que o LETRIP possuiria também maior disponibilidade de micronutrientes, pois se sabe que a disponibilidade deles diminui com a elevação do pH, exceção feita para o molibdênio (RAIJ, 1991). Em função desses maiores valores de $\mathrm{pH}$, supunha-se que o desempenho das mudas em $\mathrm{S} 1$ fosse superior ao observado em S2. Entretanto, de uma maneira geral, o cultivo em S2 proporcionou às mudas de Schinus terebinthifolius ganhos significativos no crescimento vegetativo (Tabela 2) e nas condições nutricionais (Tabela 3), em comparação com S1.

Tabela 2. Médias de altura, diâmetro do colo, matéria seca da parte aérea (MSPA), matéria seca da raiz (MSR) e colonização micorrízica de mudas de Schinus terebinthifolius Raddi., 120 dias após a semeadura ${ }^{1}$.

Table 2. Shoot height, collar diameter, shoot dry matter (MSPA) averages, root dry matter (MSR) and mycohrrizal colonization in Schinus terebinthifolius Raddi. seedlings, 120 days after sowing ${ }^{1}$.

\begin{tabular}{lccccc}
\hline Tratamento & $\begin{array}{c}\text { ALT* } \\
(\mathbf{c m})\end{array}$ & $\begin{array}{c}\text { DIAM* } \\
(\mathbf{m m})\end{array}$ & $\begin{array}{c}\text { MSPA* } \\
\left(\mathbf{g} \cdot \mathbf{p l a n t a}^{-\mathbf{1}}\right)\end{array}$ & $\begin{array}{c}\text { MSR* }^{*} \\
\left(\mathbf{g}^{\left.-\mathbf{p l a n t a}^{-1}\right)}\right.\end{array}$ & $\begin{array}{c}\text { COLONRAIZ* } \\
(\%)\end{array}$ \\
\hline S1" sem FMA & $11,0 \mathrm{~b}$ & $2,74 \mathrm{~b}$ & $0,68 \mathrm{~b}$ & $0,26 \mathrm{~b}$ & $34,6 \mathrm{~b}$ \\
S2" sem FMA & $13,2 \mathrm{a}$ & $3,96 \mathrm{a}$ & $1,14 \mathrm{a}$ & $0,55 \mathrm{a}$ & $0 \mathrm{c}$ \\
S1" com FMA & $11,2 \mathrm{~b}$ & $2,56 \mathrm{~b}$ & $0,62 \mathrm{~b}$ & $0,20 \mathrm{~b}$ & $99,6 \mathrm{a}$ \\
S2" com FMA & $12,8 \mathrm{a}$ & $3,69 \mathrm{a}$ & $1,01 \mathrm{a}$ & $0,36 \mathrm{a}$ & $0 \mathrm{c}$ \\
\hline
\end{tabular}

${ }^{1}$ Valores médios de vinte e quatro repetições. Médias seguidas de letras distintas, na coluna, diferem pelo teste de Scott-Knott (p <0,05). * ALT: altura; DIAM: diâmetro do caule; MSPA: massa seca de parte aérea; MSR: massa seca de raiz; COLONRAIZ: colonização micorrízica da raiz. "S1: substrato à base de LETRIP; S2: substrato à base de esterco bovino.

Tabela 3. Médias de teor de N, P e K (\%) na parte aérea e na raiz de mudas de Schinus terebinthifolius Raddi., 120 dias após a semeadura ${ }^{1}$.

Table 3. N, P and K (5) contents average in shoot and root of Schinus terebinthifolius Raddi. seedlings, 120 days after sowing ${ }^{1}$.

\begin{tabular}{lcccccc}
\hline Tratamento & TEORNPA* & TEORNR* & TEORPPA* & TEORPR* & TEORKPA* & TEORKR* \\
\hline S1" sem FMA & $1,38 \mathrm{~b}$ & $0,43 \mathrm{~b}$ & $0,01 \mathrm{~b}$ & $0,01 \mathrm{a}$ & $0,13 \mathrm{~b}$ & $0,05 \mathrm{a}$ \\
S2" sem FMA & $3,24 \mathrm{a}$ & $0,77 \mathrm{a}$ & $0,02 \mathrm{a}$ & $0,02 \mathrm{a}$ & $0,45 \mathrm{a}$ & $0,05 \mathrm{a}$ \\
S1" com FMA & $1,56 \mathrm{~b}$ & $0,27 \mathrm{~b}$ & $0,01 \mathrm{~b}$ & $0,00 \mathrm{~b}$ & $0,13 \mathrm{~b}$ & $0,03 \mathrm{~b}$ \\
S2" com FMA & $2,74 \mathrm{a}$ & $0,48 \mathrm{a}$ & $0,02 \mathrm{a}$ & $0,01 \mathrm{a}$ & $0,40 \mathrm{a}$ & $0,11 \mathrm{a}$ \\
\hline
\end{tabular}

${ }^{1}$ Valores médios de vinte e quatro repetições. Médias seguidas de letras distintas, na coluna, diferem pelo teste de Scott-Knott ( $\mathrm{p}$ $<0,05)$ * TEORNPA: teor de nitrogênio da parte aérea; TEORNR: teor de nitrogênio da raiz; TEORPPA: teor de fósforo da parte aérea; TEORPR: teor de fósforo da raiz; TEORKPA: teor de potássio da parte aérea; TEORKR: teor de potássio da raiz. " S1: substrato à base de LETRIP; S2: substrato à base de esterco bovino.

Uma possível explicação para os resultados encontrados seria decorrente das características físicas do LETRIP, que podem ter contribuído para o menor desempenho das mudas de Schinus terebinthifolius em S1, já que visualmente o LETRIP apresentou uma granulometria mais fina do que o esterco bovino, o que pode ter desfavorecido a dinâmica de aeração/retenção de água para as plantas em S1. Ainda existe a possibilidade de que, durante a condução do experimento, tenha ocorrido maior lixiviação de cátions em S1 do que em S2. Para que possam ser feitas maiores inferências a respeito do efeito do LETRIP e do esterco bovino na produção de mudas de Schinus terebinthifolius, é necessário que sejam realizados outros estudos.

Schinus terebinthifolius é uma espécie florestal altamente responsiva à adubação com esterco bovino curtido. Em um estudo realizado para acompanhar o desempenho de nove espécies arbóreas plantadas em uma área degradada em Itutinga, MG, durante 36 meses, Schinus terebinthifolius foi a que mais se beneficiou com a adição de esterco, tendo apresentado ganhos de $48 \%$ na altura e de $93 \%$ na área da copa (FARIA et al., 1997).

Em ambos os substratos testados, as plantas micorrizadas não apresentaram um desempenho significativamente superior ao das não-micorrizadas. Possivelmente, esse resultado foi reflexo da elevada disponibilidade de nutrientes em $\mathrm{S} 1$ e em S2. A adição de matéria orgânica ao substrato favorece o desenvolvimento de micorriza (CARNEIRO, 1995), mas isso se dá apenas sob baixas concentrações da mesma (CAPRONI, 2001), pois, sob elevada disponibilidade de nutrientes, o fungo se 
torna um encargo energético para a planta, sem o devido benefício, e a planta não-micorrizada pode ser capaz de absorver fósforo tanto quanto a micorrizada (SAGIN JÚNIOR; SILVA, 2005), podendo-se até mesmo chegar ao ponto em que a micorrização prejudica as plantas (FLORES-AYLAS et al., 2003).

A inoculação de mudas de Carica papaya L. (mamoeiro) produzidas em substrato à base de esterco, com Glomus etunicatum, somente foi positiva para o crescimento das plantas até a dose de $10 \%$ de esterco. A partir da dose de $30 \%$, a colonização radicular sofreu uma significativa redução, mostrando que, dependendo da dose de adubo aplicada, ele pode interferir negativamente nos efeitos da micorrização (TRINDADE et al., 2000). Na verdade, alguns trabalhos atestam que o elevado teor de fósforo no substrato impede que a inoculação de mudas traga benefício, como foi constatado para Schinus terebinthifolius (CARMO et al., 2004; FLORES-AYLAS et al., 2003), Mimosa artemisiana Heringer \& Paula (jurema-branca) (MOREIRA et al., 2004; SIBINEL, 2003), Psidium guajava L. (goiabeira) (SCHIAVO; MARTINS, 2002) e Hancornia speciosa Gomes (mangabeira) (COSTA et al., 2005).

Todavia, em condições de limitação nutricional, como é o caso dos solos arenosos de restinga, a micorriza pode ser importante para a ciclagem e para o estabelecimento de plantas. Em um estudo nas dunas primárias da Restinga de Barra de Maricá, RJ, verificou-se que o conteúdo de fósforo assimilável nos solos superficiais foi inferior a $1 \mathrm{mg} \cdot \mathrm{kg}^{-1}$, enquanto que na vegetação, que apresentou $100 \%$ das espécies avaliadas com micorriza, a concentração desse nutriente variou entre 100 e 1.000 ppm, o que enfatizou a eficiência dessa simbiose nesse ecossistema (HAY; LACERDA, 1984).

Para atender à demanda de programas de recuperação de áreas degradadas por mudas de espécies florestais, a Embrapa Agrobiologia lança mão da mistura de propágulos acima citada. Essa medida visa potencializar os benefícios da micorrização para a planta hospedeira, uma vez que cada espécie de FMA contribuiria com diferentes estratégias de colonização radicular, sob um amplo espectro de condições ambientais e com uma maior variabilidade da capacidade de difusão e multiplicação, já que um único fungo não apresentaria uma ampla gama de características desejáveis (CAPRONI et al., 2005).

No presente estudo, inicialmente, outra variável seria analisada: a densidade de esporos presente no substrato. Porém, durante a contagem de esporos, verificou-se que tanto S1 sem quanto S1 com inoculação apresentaram abundantes estruturas de um material elastômero, as quais muito se assemelhavam a esporos de fungos micorrízicos arbusculares, além da presença de alguns esporos de FMA. Portanto, optou-se por não considerar os resultados dessa variável, com o intuito de evitar a obtenção de resultados superestimados.

Não houve correlação direta entre a COLONRAIZ e a resposta da planta em benefícios vegetativos e nutricionais. Assim sendo, o presente estudo está de acordo com Caldeira et al. (1999), os quais verificaram que o tratamento com Gigaspora margarita, mesmo tendo ocasionado a menor porcentagem do comprimento de raízes colonizadas, foi superior ou equivalente à testemunha (sem inoculação) quanto à altura, ao diâmetro e à massa seca de raízes finas e grossas de Peltogyne venosa (Vahl) Benth (pau-roxo-da-várzea) e Sclerolobium paniculatum Vogel (taxi-branco).

Não foi observada colonização de raiz no tratamento sem FMA, o que reforça a efetividade do fungicida usado para a desinfestação do substrato. Contudo, essa variável atingiu valor considerável em S1 sem FMA $(34,6 \%)$, o que poderia levar a crer que houve uma contaminação das mudas nesse tratamento, apesar dos cuidados tomados (disposição das plantas dos tratamentos com e sem inóculo em bancadas separadas; controle, por meio da utilização de estopas embebidas com óleo queimado nas pernas das bancadas, das formigas, as quais poderiam transportar propágulos de fungos micorrízicos; irrigação muda a muda, com água destilada e auxílio de pissete, evitando a formação de sprays (aerossóis), que poderiam contaminar os tratamentos não inoculados). Quanto a essa variável, também destaca-se a disparidade observada entre S2 com e S1 com, já que a elevada disponibilidade de nutrientes só poderia justificar a ausência de colonização de raízes em S2 com, o que não foi verificado em S1 com, onde a média de COLONRAIZ foi de aproximadamente 100\% (Tabela 2). Portanto, houve a necessidade de investigar mais detalhadamente o motivo de tal resultado.

Dessa maneira, após submeter os componentes individualizados de S1 e S2 à marcha de extração de esporos, a análise em microscópio estereoscópico constatou a ausência de esporos de FMA na areia, na argila e no esterco, os quais estiveram presentes no LETRIP (dados não incluídos, provenientes de avaliação qualitativa). A partir daí, procedeu-se ao fracionamento das partículas do 
LETRIP com o auxílio de estiletes, o que permitiu observar, por meio de visualização em lupa, que delas partiam esporos e hifas de FMA. Em seguida, para análise do material em microscópio modelo Standard 25, fabricante Zeiss, sob aumento de 10 vezes, foi montada uma lâmina em água com essas partículas, cujas imagens foram retratadas por meio de máquina digital Olympus, com recurso próximo de aproximação (zoom digital de 4 vezes), como pode ser observado na figura 1.

Acredita-se que as partículas de borracha sintética componentes do LETRIP permitiram o encapsulamento dos esporos dos FMA nativos presentes em tal material, protegendo-os contra o fungicida empregado, os quais, após efeito do fungicida ter se tornado inócuo, iniciaram a colonização das raízes. Essa hipótese explicaria a colonização radicular em S1 sem FMA, enquanto o altíssimo percentual de colonização de raiz das mudas produzidas em S1 com FMA poderia ter sido fruto de um efeito sinérgico entre os FMA nativos no LETRIP e aqueles empregados no inóculo. Dessa maneira, foi descartada a possibilidade de contaminação das mudas não inoculadas em S1, na casa de vegetação.

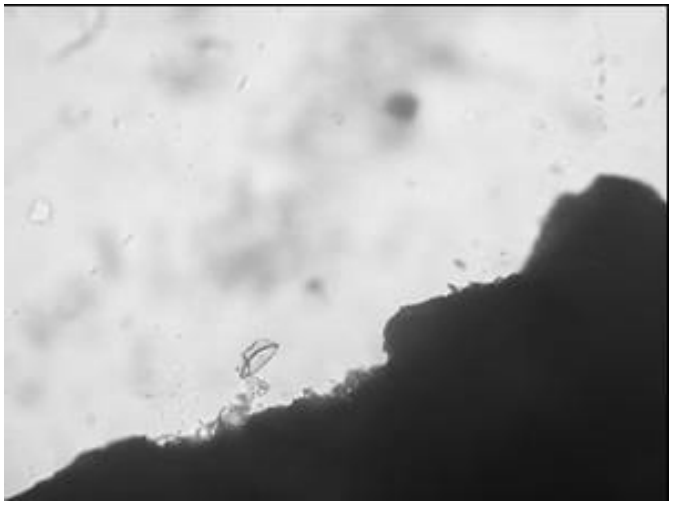

(a)

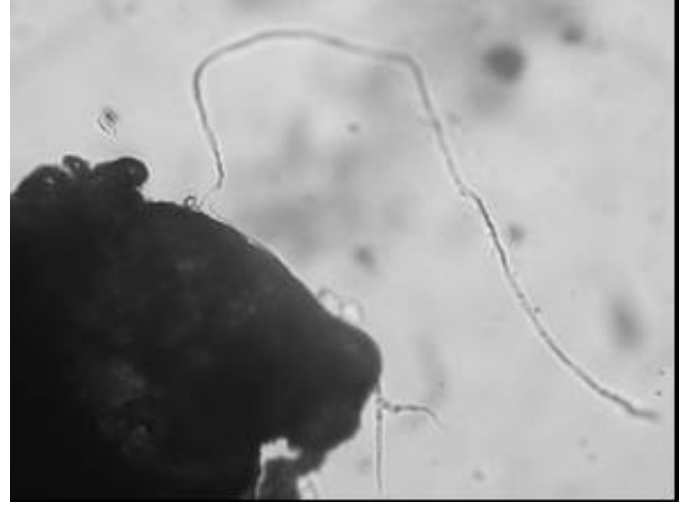

(b)

Figura 1. Detalhe de esporo de FMA ligado a partícula de borracha do LETRIP (a); hifas de FMA presas à partícula de borracha do LETRIP (b).

Figure 1. AMF spore attached to a LETRIP rubber particle (a); AMF hyphae attached to a LETRIP rubber particle (b).

Comparando-se o efeito do LETRIP e o do esterco bovino curtido como constituintes de substrato para a produção de mudas de Schinus terebinthifolius, embora o primeiro material tenha apresentado maiores teores disponíveis de $\mathrm{Ca}, \mathrm{Mg}$ e $\mathrm{P}$ e valor de $\mathrm{pH}$ mais favorável à disponibilidade de micronutrientes para as plantas, o substrato à base de esterco (S2) empregado no presente estudo foi quem promoveu ganhos significativamente superiores às plantas. Então, para preencher lacunas relacionadas ao efeito desse material como condicionador de substrato, é importante que sejam realizados outros estudos, os quais poderiam estimular o uso alternativo do LETRIP e contribuir para destinar a esse resíduo industrial uma disposição final ambientalmente mais adequada. Nesse sentido, outras espécies florestais poderiam ser cultivadas em substratos contendo diferentes proporções de LETRIP. Outra questão que merece ser investigada é a efetividade de variadas dosagens de diferentes fungicidas e da autoclavagem na eliminação de propágulos dos fungos presentes no LETRIP, para que se esclareçam os mecanismos pelos quais esse resíduo desempenhou um papel "protetor" dos fungos nativos contra a ação do fungicida empregado no presente estudo.

\section{CONCLUSÕES}

O substrato S2, composto à base de esterco bovino curtido, é o recomendado para a produção de mudas de Schinus terebinthifolius, uma vez que conduziu a ganhos significativos das plantas em altura, diâmetro, massa seca de parte aérea e de raiz, teor de N, P e K na parte aérea, e de N na raiz, em comparação com S1, à base de LETRIP. 
A inoculação das mudas de Schinus terebinthifolius com fungos micorrízicos arbusculares não proporcionou benefícios a elas, provavelmente devido ao elevado teor de nutrientes nos substratos testados.

Outros estudos devem ser realizados para que se esclareçam os mecanismos pelos quais o LETRIP “protegeu” os fungos nativos contra a ação do fungicida empregado na desinfestação dos substratos testados.

\section{REFERÊNCIAS}

ARAÚJO, D. S. D. Análise florística e fitogeográfrica das Restingas do Estado do Rio de Janeiro. Tese (Doutorado em Ecologia) - Instituto de Biologia, Universidade Federal do Rio de Janeiro, Rio de Janeiro, 2000.

CALDEIRA, M. V. W.; SILVA, E. M. R., FRANCO, A. A.; ZANON, M. L. B. Efeito de fungos micorrízicos arbusculares no desenvolvimento de duas leguminosas arbóreas. Ciência Florestal, Santa Maria, RS, v. 9, n. 1, p. 63-70. 1999.

CAPRONI, A. L. Fungos micorrízicos arbusculares em áreas reflorestadas remanescentes da mineração de bauxita em Porto de Trombetas/PA. 205 f. Tese (Doutorado em Agronomia) Universidade Federal Rural do Rio de Janeiro, Seropédica, 2001.

CAPRONI, A. L.; FRANCO, A. A.; BERBARA, R. L. L.; GRANHA, J. R. D. O.; MARINHO, N. F. Fungos micorrízicos arbusculares em estéril revegetado com Acacia magium, após mineração de bauxita. Revista Árvore, Viçosa, MG, v. 29, n. 3, p. 373-381, 2005.

CARMO, M. V.; LOUREIRO, D. C.; OLIVEIRA NETO, S. N.; LELES, P. S. S.; SILVA, E. M. R. Avaliação de recipientes para produção de mudas e efito da associação micorrízica em Schinus terebinthifolius Raddi. In: JORNADA DE INICIAÇÃO CIENTÍFICA DA UFRuralRJ, Seropédica, RJ, 14., 2004, Seropédica. Anais... Seropédica: Universidade Federal Rural do Rio de Janeiro, 2004. v. 14, n. 1, p. 273-276. Área de Recursos Florestais e Engenharia Florestal.

CARNEIRO, J. G. A. Produção e controle de qualidade de mudas florestais. Curitiba: UFPR/FUPEF; Campos: UENF, 1995. 451 p.

CARNEIRO, M. A. C.; SIQUEIRA; J. O.; MOREIRA, F. M. S.; CARVALHO, D.; BOTELHO, S. A.; SAGGIN JÚNIOR, O. J. Micorriza arbuscular em espécies arbóreas e arbustivas nativas de ocorrência no sudeste do Brasil. Cerne, Lavras, v. 4, n. 1, p. 129-145, 1998.

CARRASCO, P. G.; CASTANHEIRA, S. A. Recipientes e substratos na produção de mudas de espécies florestais de Restinga em Ilha Comprida, SP. Arquivos do Instituto de Biologia, [S.1.], v. 71, p. 305-307, 2004. Suplemento.

CARVALHO, P. E. R. Espécies arbóreas brasileiras. Brasília, DF: Embrapa Informação Ecológica; Colombo: EMBRAPA Florestas, 2003. p. 161-168.

CARVAlHO FIlHO, J. L. S.; ARRIGONI-BlANK, M. F.; BLANK, A. F.; RANGEL, M. S. A. Produção de mudas de jatobá (Hymenaea courbaril L.) em diferentes ambientes, recipientes e composições de substratos. Cerne, Lavras, v. 9, n. 1, p. 109-118, 2003.

COSTA, C. M. C.; CAVAlCANTE, U. M. T.; GOTO, B. T.; SANTOS, V. F.; MAIA, L. C. Fungos micorrízicos arbusculares e adubação fosfatada em mudas de mangabeira. Pesquisa Agropecuária Brasileira, Brasília, DF, v. 40, n. 3, p. 225-232, 2005.

CUNHA, A. O.; ANDRADE, L. A.; BRUNO, R. L. A.; SILVA, J. A. L.; SOUZA, V. C. Efeitos de substratos e das dimensões dos recipientes na qualidade das mudas de Tabebuia impetiginosa (Mart. ex D. C.) Standl. Revista Árvore, Viçosa, MG, v. 29, n. 4, p. 507-516, 2005.

DIAS, F. C. Dinâmica e disponibilidade de nutrientes e metais pesados em área de planossolo tratado com resíduos industriais. 77 f. Dissertação (Mestrado em Agronomia) - Instituto de Agronomia, Universidade Federal Rural do Rio de Janeiro, Seropédica, 2005. 
DIAS, F. C.; BEHLING, M.; OLIVEIRA, J. A.; MELO, M. P.; MAZUR, N.; AMARAL SOBRINHO, N. M.; OLIVEIRA, C. Alterações da acidez do solo tratado com resíduos industriais. In: CONGRESSO DE PESQUISA CIENTÍFICA DA UFRuralRJ, 2., 2004, Seropédica, RJ. Anais... Seropédica: Universidade Federal Rural do Rio de Janeiro, 2004. v. 2, n. 1, p.11-15. Área de Agronomia.

EMPRESA BRASILEIRA DE PESQUISA AGROPECUÁRIA (EMBRAPA). Centro Nacional de Pesquisas em solos. Manual de métodos de análise de solos. Rio de Janeiro, 1997. 212 p.

FARIA, J. M. R.; DAVIDE, A. C.; BOTELHO, S. A. Comportamento de espécies florestais em área degradada, com duas adubações de plantio. Cerne, v. 3, n. 1, 1997. Disponível em: <http://www.dcf.ufla.br/CERNE/revistav3n1-1997/cemig.PDF>. Acesso em: 13/03/2005.

FRANCO, A. A.; CAMPELlO, E. F.; SILVA, E. M. R.; FARIA, S. M. Revegetação de solos degradados. Seropédica: Empresa Centro Nacional de Pesquisa de Agrobiologia (EMBRAPACNPAB), 1992.8 p. (Comunicado Técnico, n. 9).

FREIRE, L. R.; BLOISE, R. M.; MOREIRA, G. N. C.; EIRA, P. A. Análise química do solo. In: DEPOLLI, H. (Coord.); ALMEIDA, D. L.; SANTOS, G. A.; CUNHA, L. C.; FREIRE, L. R.; AMARAL SOBRINHO, N. M. B.; PEREIRA, N. N. C.; EIRA, P. A.; BLOISE, R. M.; SALEK, R. C. Manual de adubação para o Estado do Rio de Janeiro. Itaguaí, RJ: Editora Universidade Rural, 1988. p.24-37. (Coleção Universidade Rural. Ciências Agrária, n. 2).

GERDEMANN, J. W.; NICOLSON, T. H. Spores of mycorrhizal endogone species extracted from soil by wet sieving and decanting. Transactions of British Mycological Society, [S.1.], v. 46, p. 235-244, 1963.

GIOVANETTI, M.; MOSSE, B. An evaluation of techniques for measuring vesicular-arbuscular mycorrhizal infection in roots. New Phytologist, New York, v. 64, n. 3, p. 489-500, 1980.

GONÇALVES, J. L. M.; NOGUEIRA JÚNIOR, L. R.; DUCATTI, F. Recuperação de solos degradados. In: KAGEYAMA, P. Y.; OLIVEIRA, R. E.; MORAES, L. F. D.; ENGEL, V. L.; GANDARA, F. B. (Orgs.). Restauração ecológica de ecossistemas naturais. Botucatu: FEPAF, 2003. p. 111-163.

GRACE, C.; STRIBLEY, D. P. A safer procedure for routine staining of vesicular-arbuscular mycorrhizal fungi. Mycological Research, Cambridge, v. 95, p. 1160-1162, 1991.

HAY, J. D.; LACERDA, L. D. Ciclagem de nutrientes do ecossistema de Restinga. In: LACERDA, L. D.; ARAÚJO, D. S. D.; TURCQ, B. (Orgs.) Restingas: origens, estrutura, processos. Niterói: CEUFF, 1984. p. 459-475.

HUNGRIA, M.; ARAÚJO, R. S. (Eds.). Manual de métodos empregados em estudos de microbiologia agrícola. EMBRAPA: Brasília, DF, 1994. 525 p.

JESUS, R. M.; ROLIM, S. G. Experiências relevantes na restauração da Mata Atlântica. In: GALVÃO, A. P. M.; PORFÍRIO-DA-SILVA, V. (Eds.). Restauração Florestal: fundamentos e estudos de caso. Colombo: EMBRAPA Florestas, 2005. p. 59-86.

KAGEYAMA, P. Y.; GANDARA, F. B. Resultados do programa de restauração com espécies arbóreas nativas do convênio ESALQ / USP e CESP. In: GALVÃO, A. P. M.; PORFÍRIO-DA-SILVA, V. (Eds.). Restauração Florestal: fundamentos e estudos de caso. Colombo: EMBRAPA Florestas, 2005. p. 47-58.

KAHILUOTO, H.; VESTBERG, M. Creation of a non-mycorrhizal control for a bioassay of AM: Benomyl application and soil sampling time. Mycorrhiza, [S.1.] , n. 9, p. 259-270, 2000.

KOSKE, R. E.; GEMMA, J. N. A modified procedure for staining roots to detect VA mycorrhizas. Mycological Research, Cambridge, v. 92, p. 486-488, 1989. 
LENZI, M.; ORTH, A. I. Fenologia reprodutiva, morfologia e biologia floral de Schinus terebinthifolius Raddi. (Anacardiaceae), em restinga da Ilha de Santa Catarina, Brasil. Biotemas, Florianópolis, v. 17, n. 2, p. 67-89, 2004.

LORENZI, H. Árvores brasileiras: manual de identificação e cultivo de plantas arbóreas nativas do Brasil. 3. ed. Nova Odessa: Plantarum, 2000. v. 1.

MALAVOLTA, E.; BOARETTO, A. E.; PAULINO, V. T. Micronutrientes: uma visão geral. In: FERREIRA, M. E.; CRUZ, M. C. P. (Eds.). Micronutrientes na agricultura. Piracicaba: POTAFOS/CNPq, 1991. p. 01-33.

MALHEIROS, M. G.; FREITAS, F. C.; FRADE JÚNIOR, E. F.; LYRIO, J. J.; OLIVEIRA NETO, S. N.; LELES, P. S. S.; LIMA, E.; ZONTA, E.; AMARAL SOBRINHO, N. M. B. Resíduo industrial classe II na produção de mudas de eucalipto. In: JORNADA DE INICIAÇÃO CIENTÍFICA DA UFRuralRJ, 15., 2005, Seropédica, RJ. Anais... Seropédica: Universidade Federal Rural do Rio de Janeiro, 2005. 1 CD-ROM.

MENEZES, L. F. T; ARAÚJO, D. S. D.; GOES, M. H. B. Marambaia: a última restinga preservada. Ciência Hoje, São Paulo, v. 23, n. 136, p. 28-37, 1998.

NEWMAN, E. I. A method of estimating the total length of root in a sample. Journal of Applied Ecology, Oxford, v. 3, n. 2, p.139-145, 1966.

ODUM, E. P. Ecologia. Rio de Janeiro: Guanabara Koogan, 1988. 434p.

RAIJ, B. Fertilidade do solo e adubação. Piracicaba: Ceres/Potafos, 1991. 343 p.

RESENDE, A. V.; FURTINI NETO, A. E.; MUNIZ, J. A.; CURI, N.; FAQUINI, V. crescimento inicial de espécies florestais de diferentes grupos sucessionais em resposta a doses de fósforo. Pesquisa Agropecuária Brasileira, Brasília, DF, v. 34, n. 11, p.2071-2081, 1999.

RIZZINI, C. T. Tratado de fitogeografia do Brasil. Aspectos morfológicos, sociológicos e florísticos. 2. ed. Rio de Janeiro: Âmbito Cultural,1997. 746 p.

SCHIAVO, J. A.; MARTINS, M. A. Produção de mudas de goiabeira (Psidium guajava L.), inoculadas com o fungo micorrízico arbuscular Glomus clarum, em substrato agro-industrial. Revista Brasileira de Fruticultura, Cruz das Almas, v. 24, n. 2, p. 519-523, 2002.

SIBINEL, A. H. M. Resposta da leguminosa Mimosa artemisiana Heringer \& Paula à inoculação de diferentes fungos micorrízicos arbusculares na recuperação de áreas degradadas. 60 f. Dissertação (Mestrado em Agronomia) - Instituto de Agronomia, Universidade Federal Rural do Rio de Janeiro, Seropédica, 2003.

SOUZA, F. A.; SILVA, E. M. R. Micorrizas arbusculares na revegetação de áreas degradadas. In: SIQUEIRA, J. O. (Ed.) Avanços em fundamentos e aplicação de micorrizas. Lavras: UFLA/DCS e DCF, 1996. p. 253-290.

TEDESCO, J.; GIANELLO, C.; BISSANI, C. A.; BOHNEN, H.; VOLKWEISS, S. J. Análises de solo, planta e outros materiais. Porto Alegre: UFRGS, 1995. 174 p.

TRINDADE, A. V.; FARIA, N. G.; ALMEIDA, F. P. Uso de esterco no desenvolvimento de mudas de mamoeiro colonizadas com fungos micorrízicos. Pesquisa Agropecuária Brasileira, Brasília, DF, v. 35, n. 7, p. 1389-1394, 2000. 